\title{
Increased chiasma frequency as a result of chromosome rearrangement
}

\author{
J. S. Parker
}

School of Biological Sciences, Queen Mary College, Mile End Road, London E1 4NS, U.K.

\begin{abstract}
The effects of structural chromosome changes on chiasma frequency have been studied in a single family of the plant Hypochoeris radicata $2 n=8$ segregating for two rearrangements: fission of chromosome 1 and interchange between chromosomes 1 and 2 . In the family seven karyotypically-distinct groups of offspring can be distinguished which has enabled comparisons of chiasma effects to be made independent of genetic background. Both fission and interchange heterozygotes have an increase of about 0.5 chiasmata per PMC over basic homozygotes and these effects are simply additive. Increases in chiasma frequency are directly correlated with increases in the number of potentially-paired segments in the complement at pachytene. Comparisons of interchange heterozygotes with and without centric fission show that the chiasma increase is entirely restricted to the segments on either side of the structural rearrangement break-point. It is argued that chiasma modification is likely to result from changes in pairing patterns rather than interference reduction in the vicinity of the break-point.
\end{abstract}

\section{INTRODUCTION}

Interbivalent effects of structural rearrangements on cross-over numbers and positions are well known in Drosophila, and inversions are routinely employed in genetical investigations to increase recombination in specific regions of the genome (Lucchesi, 1976). Despite the wealth of cytological studies of structural rearrangements little comparable recombination data exist at the chiasma level (Sybenga, 1975). In a recent study, clear chiasma effects of both centric fission and interchange were demonstrated in the plant Hypochoeris radicata (Parker et al., 1982). In families of this species segregating for structural rearrangements heterozygotes regularly formed more chiasmata than their structurally-normal full sibs. A relationship was demonstrated between the number of additional potentially-paired segments at pachytene generated by rearrangement and the extent of the chiasma increase. A single additional segment, whether resulting from interchange or centric fission, raised the chiasma frequency by 0.45 and a pair of segments by 0.9 chiasmata per PMC.

In $H$. radicata only one of the four bivalents can be identified at metaphase-I of meiosis when chiasma number and position can be most accur- ately determined (Parker, 1975). It was not possible in the previous study to localise the chiasma increase within the complement precisely although it was inferred that chiasma increase was associated predominantly with the structurallyrearranged chromosomes (Parker et al., 1982). In this present paper chiasma analysis of a family segregating for two different structural changescentric fission of chromosome 1 and interchange between chromosomes 1 and 2-is presented. Testing two structural changes in a single segregating family has demonstrated the following:

(a) it is not fortuitous that the amount of the chiasma increase attributable to particular rearrangements is identical;

(b) the "excess" chiasmata generated by the rearrangements not only occur in the structurallyrearranged chromosomes but are localised to the chromosome segments on either side of the break points.

Interestingly, no interchromosomal effects associated with either rearrangement have been detected.

\section{MATERIALS AND METHODS}

A single family of Hypochoeris radicata comprising 76 offspring was studied at metaphase-I of meiosis. 
The parents used in the cross were chosen from two experimental lines and shared a single common grandparent. The structural rearrangements themselves occurred spontaneously in unrelated experimental lines five (interchange) and six (centric fission) generations before the cross was made. $H$. radicata is strictly self-incompatible and crosses were made without masculation by rubbing fullyopen capitula together.

Capitula (buds) were fixed in Carnoy's fixative and individual florets were squashed in $2 \%$ lactopropionic orcein. In each plant $40 \mathrm{PMCs}$ at metaphase-I were scored both for chiasma number and for chiasma position as accurately as the karyotype would allow.

\section{The segregating family}

Hypochoeris radicata $(2 n=8)$ has three pairs of acrocentric chromosomes of similar size (1-3) and a smaller metacentric pair (4). Arm ratios of the acrocentrics are about $1: 2$ and the slightly smaller pair 3 carries a nucleolar-organiser region terminal on the short arm (fig. 1).

The structural changes used in this analysis were: (i) centric fission of chromosome 1 (fig. 2), (ii) a reciprocal interchange between the long arm of chromosome 1 and short arm of chromosome 2 . The interchange is highly unequal giving a small $\left(1^{2}\right)$ and a large (2) metacentric (fig. 3). In heterozygotes chiasmata do not form in one pairing segment and the pachytene association is effectively three-armed.

The cross was made using an interchange heterozygote as egg parent. The pollen parent carried the interchange and in addition the non- interchanged chromosome 1 was present as two telocentric elements (figs. 2 and 3). Two balanced gametes are produced by the egg parent and four by the pollen parent (fig. 8). The two additional gametic types in the pollen result from the occurrence of chiasmata in the region between the two points of rearrangement, designated as segment 4 in the pachytene cross. Although eight gametic combinations occur in this cross, only seven classes of chromosomally-identifiable offspring result (figs 1-7), since interchange heterozygotes are produced in two ways (fig. 8).

In basic homozygotes of $H$. radicata chiasmata can be assigned only to the long or short arms of the acrocentric group (1-3) or to pair 4 . In structural heterozygotes more precise chiasma location is possible. In $1 / 2$ interchange heterozygotes chiasmata can be assigned to six potentially-chiasmate regions within the pachytene cross, the long or short arms of pair 3, and to pair 4. This applies also to interchange heterozygotes with telocentric elements. In fission heterozygotes chromosomes 2 and 3 are not distinguishable but 1 and 4 are. Chromosome 4 can thus be identified in all karyotype classes except interchange homozygotes in which pair $1^{2}$ has the same morphology. In this family, then, chiasma position was recorded as accurately as the karyotype allows.

\section{RESULTS}

\section{Variation in mean chiasma frequency within the family}

Mean chiasma frequencies in the 76 full sibs ranged from $4 \cdot 300$ chiasmata per PMC to 6.600 (table 1 ).

Table 1 Chiasma frequencies of the seven morphologically-distinguishablc karyotype classes segregating in the offspring of a cross in Hypochoeris radicata between two interchange heterozygotes, one carrying telocentric elements of chromosome 1 (see fig. 8 ). Within each karyotype chiasma frequencies are allotted to all identifiable chromosomes or chromosome groups

\begin{tabular}{|c|c|c|c|c|c|c|c|c|c|c|c|}
\hline \multirow[b]{2}{*}{ Karyotype } & \multirow{2}{*}{$\begin{array}{l}\text { No. } \\
\text { individuals }\end{array}$} & \multirow{2}{*}{$\begin{array}{l}\text { Mcan }+ \\
\text { range }\end{array}$} & \multicolumn{9}{|c|}{ Chiasma frequencies in identifiable chromosomes } \\
\hline & & & $1^{\mathrm{s}} 1^{2}$ & $1+2$ & $1+2+3$ & $1^{2}$ & $1^{2}+4$ & $2^{1}$ & $2+3$ & 3 & 4 \\
\hline $11^{s} 1^{L} 22$ & 11 & $\begin{array}{l}5 \cdot 395 \\
4 \cdot 825-5 \cdot 850\end{array}$ & 1.86 & - & - & - & - & - & $2 \cdot 461$ & - & 1.075 \\
\hline $11^{2} 22^{1}$ & 11 & $\begin{array}{l}5.405 \\
4.775-5.750\end{array}$ & - & $2 \cdot 907$ & - & - & - & - & - & $1 \cdot 339$ & $1 \cdot 159$ \\
\hline $1^{\mathrm{s}} 1^{\mathrm{L}} 1^{2} 22^{1}$ & 11 & $\begin{array}{l}5 \cdot 800 \\
5 \cdot 225-6 \cdot 600\end{array}$ & - & $3 \cdot 384$ & - & - & - & - & - & $1 \cdot 302$ & $1 \cdot 114$ \\
\hline $11^{2 \mathrm{~s}} 1^{2 \mathrm{~L}} 22^{l}$ & 10 & $\begin{array}{l}5 \cdot 980 \\
5 \cdot 600 \cdots 6 \cdot 500\end{array}$ & - & $3 \cdot 525$ & - & - & - & - & - & $1 \cdot 280$ & $1 \cdot 175$ \\
\hline $1^{2} 1^{2} 2^{1} 2^{1}$ & 11 & $\begin{array}{l}5 \cdot 070 \\
4 \cdot 425-5 \cdot 600\end{array}$ & - & -- & -- & - & $2 \cdot 343$ & $1 \cdot 423$ & - & $1 \cdot 270$ & - \\
\hline
\end{tabular}



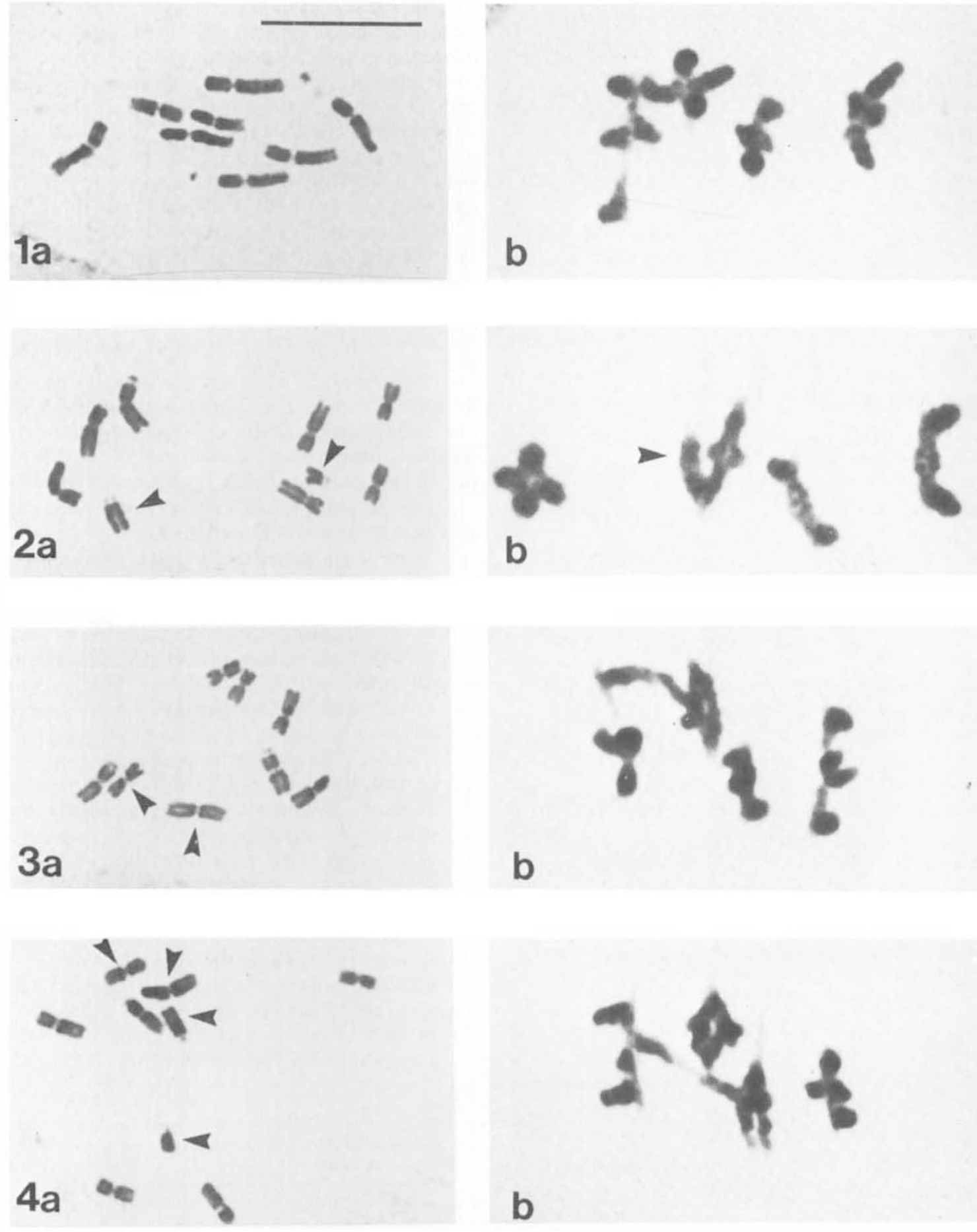

Figures 1-4 1. Mitotic (a) and meiotic (b) chromosomes of Hypochoeris radicata $2 n=8$. 2. Fission-1 heterozygote $\left(11^{\mathrm{S}} 1^{\mathrm{L}} 22\right)$ at mitosis (a) and meiosis (b) with the telotrivalent in convergent orientation. $1^{\mathrm{S}}, 1^{\mathrm{L}}$ and telotrivalent arrowed. 3. Chromosome $1 / 2$ interchange heterozygote $\left(11^{2} 22^{1}\right)$ at mitosis (a) with interchange products arrowed; (b) an association of four at metaphase-I with chiasma formation in segments $1,3,4$ and 5. 4. Fission-1+interchange $1 / 2$ heterozygote $\left(1^{\mathrm{S}} 1^{\mathrm{L}} 1^{2} 22^{1}\right)$ at mitosis (a); (b) metaphase-I with chiasma formation in segments $2,3,4$ and 5 of the multiple. $1^{\mathrm{S}}, 1^{\mathrm{L}}, 1^{2}$ and $2^{1}$ arrowed. Scale represents $10 \mu \mathrm{m}$. 

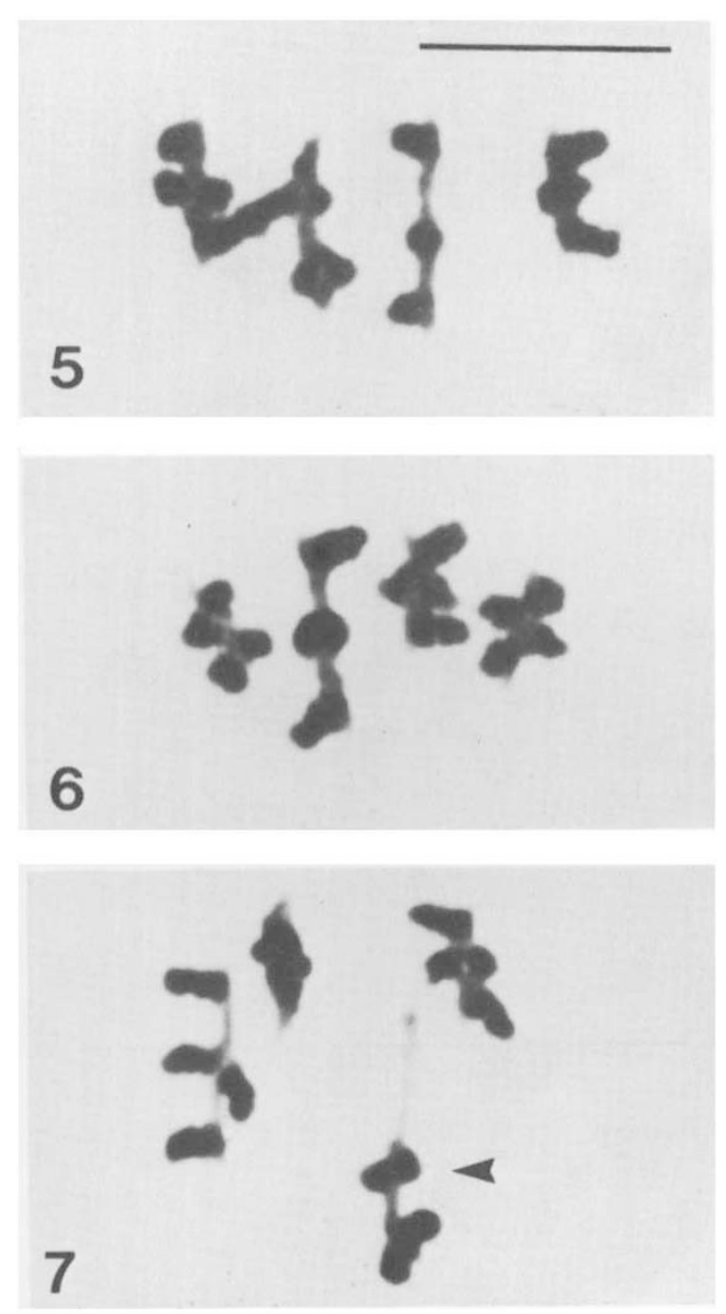

Figures 5-7 5. Fission- $1^{2}$, interchange $1 / 2$ heterozygote $\left(11^{2 S_{1}} 1^{2 \mathrm{~L}} 22^{1}\right)$ at metaphase I of meiosis; chiasma formation in segments 1,3,4 and 5 of the multiple. 6. Metaphase-I in an interchange homozygote $\left(1^{2} 1^{2} 2^{1} 2^{1}\right) .7$. Metaphase-I in an interchange homozygote with fission products of one chromosome $1^{2}\left(1^{2} 1^{2 \mathrm{~S}} 1^{2 \mathrm{~L}} 2^{1} 2^{1}\right)$; telotrivalent in linear orientation (arrowed). Scale represents $10 \mu \mathrm{m}$.

The individual plant means are distributed in an approximately normal fashion with an overall mean of 5.437 (fig. 9).

\section{Variation in mean chiasma frequency between karyotype classes}

The mean chiasma frequency assessed for each of the seven karyotypic classes ranged from 4.832 in basic homozygotes to 5.980 in interchange heterozygotes with telocentric derivatives of chromosome $1^{2}$. An analysis of variance (table 2 ) indicates that difference between classes are sig- nificant at the $1 \%$ level $\left(F_{6,69}=13 \cdot 667\right)$. Comparison of class means, examined by a StudentNewman-Keul multiple range test, shows that basic and interchange homozygotes do not differ but have significantly lower means than all the structurally heterozygous classes.

Chiasma frequencies differ considerably between individuals within classes (table 1). Despite this, two groups of full sibs show no overlap. Basic homozygotes range from $4 \cdot 300$ to $5 \cdot 225$ while interchange heterozygotes carrying $1^{2}$ as telocentrics range from $5 \cdot 600$ to $6 \cdot 500$ (fig. 9).

\section{Relationships between chromosome structure and chiasma frequency}

Two relationships between chromosome structure and mean chiasma frequency can be deduced from comparisons of class means:

(a) In the previous study the chiasma frequency increases attributable to $1 / 2$ interchange and centric fission of chromosome 1 were both about 0.5 chiasmata per PMC (Parker et al., 1982). These increases were assessed in different families so the similarity may have been fortuitous. Within this family these classes have almost identical chiasma frequencies-5-395 in fission-1 heterozygotes and 5.405 in interchange heterozygotesabout 0.56 higher than basic homozygotes. Clearly, the equivalence of these two types of structural change is a real phenomenon.

(b) Chiasma frequency increase resulting from structural rearrangements in $H$. radicata is closely correlated with the numbers of potentially-paired segments present in the pachytene complement (Parker et al., 1982). An increase of one paired segment leads to an increase of about 0.5 chiasmata per PMC. Within this family the chiasma frequencies can be assessed on the basis of the difference in number of paired segments at pachytene- 0 , 1 or 2 (table 3 ). The overall figures are $+0 \cdot 176$,

Table 2 Analysis of variance of chiasma frequencies in 76 full-sibs classified into seven karyotype groups in the segregating fanily

\begin{tabular}{|c|c|c|c|c|}
\hline Item & $\mathrm{df}$ & SS & MS & $\mathrm{F}$ \\
\hline \multicolumn{5}{|l|}{ Between } \\
\hline $\begin{array}{l}\text { karyotype } \\
\text { classes }\end{array}$ & 6 & $10 \cdot 333$ & 1.722 & $13.667^{* *}$ \\
\hline $\begin{array}{l}\text { Between } \\
\text { individuals }\end{array}$ & 69 & $8 \cdot 717$ & $0 \cdot 126$ & \\
\hline Totals & 75 & $19 \cdot 050$ & & \\
\hline
\end{tabular}




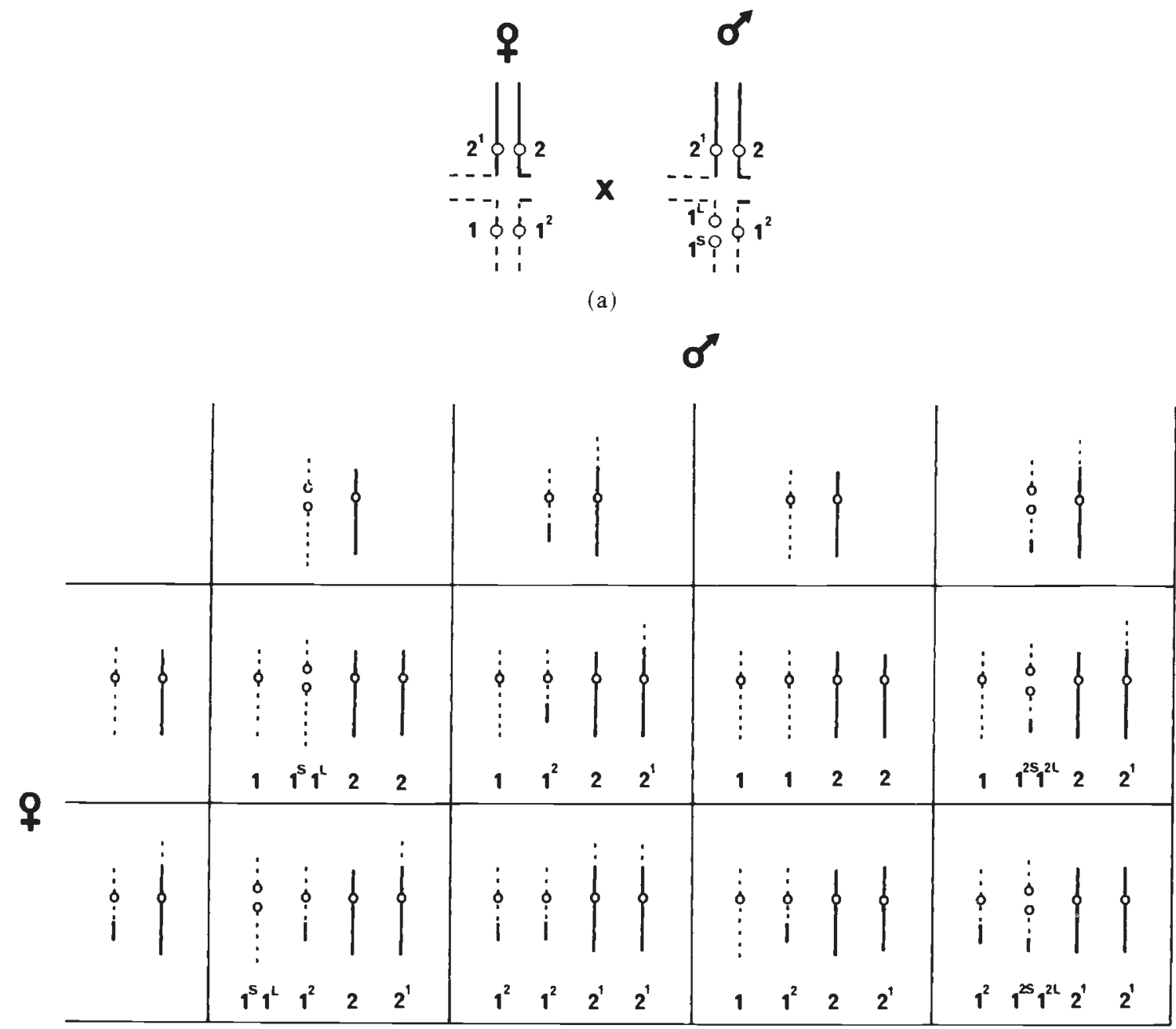

(b)

Figure 8 Diagrammatic representationn of the results of a cross between an interchange heterozygote involving chromosomes 1 and 2 of Hypochoeris radicata $\left(11^{2} 22^{1}\right)$ as egg parent and an interchange heterozygote which also carried telocentric derivatives of chromosome $1\left(1^{\mathrm{S}} 1^{\mathrm{L}} 1^{2} 22^{\mathrm{l}}\right)$ as pollen parent. The pachytene pairing configuration of the two parents are also illustrated.

Table 3 Differences in chiasma frequency between karyotype classes within the segregating family. The figures enclosed within the dashed lines are for karyotypes which differ by one pairing segment, while those within the solid line are for karyotypes differing by two pairing segments. The remaining comparisons involve no differences in pairing segments

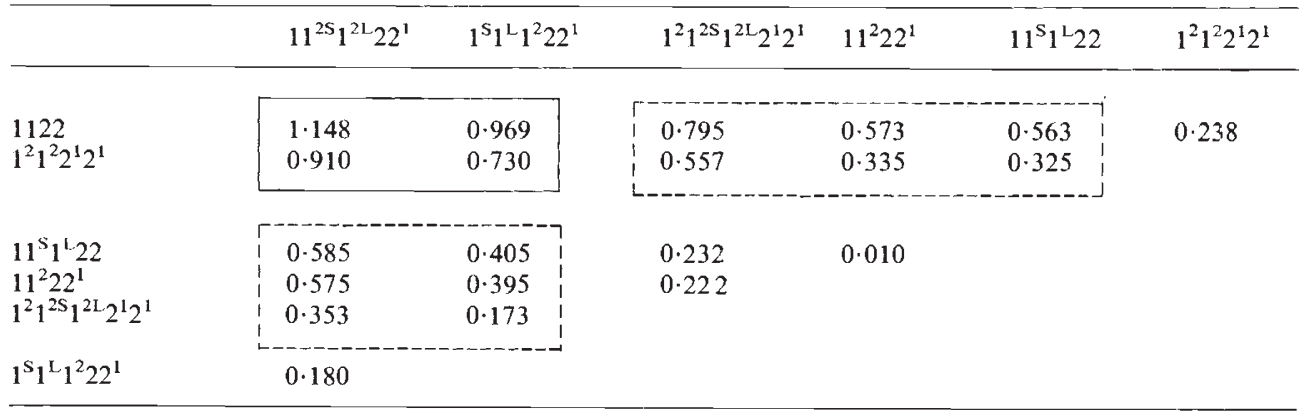




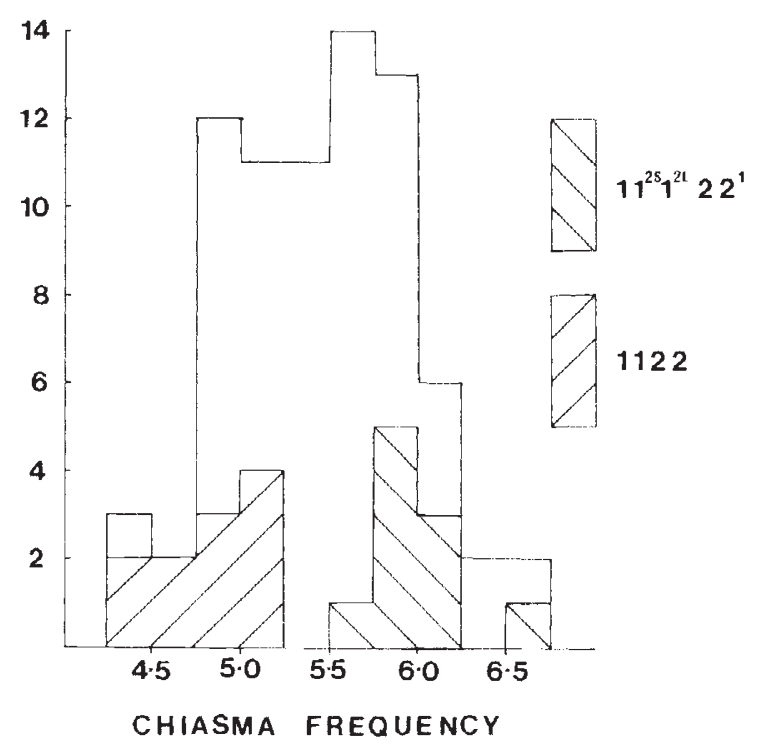

Figure 9 Histogram of mean chiasma frequencies of 76 fuli sibs of a cross between an interchange heterozygote and an interchange/fission heterozygote in Hypochoeris radicata. Mean chiasma frequencies of two structurallydistinct groups of sibs (1122 and $\left.11^{2 \mathrm{~S}} 1^{2 \mathrm{~L}} 22^{\mathrm{l}}\right)$ which show no overlap are indicated.

+0.456 and +0.939 chiasmata for differences of 0,1 and 2 segments respectively. The results obtained previously between families are borne out by within-family comparison.

\section{Localisation of chiasma increase within the complement}

In those karyotype classes carrying interchange $1 / 2$ or its derivatives (five out of seven) pai 3 can be unequivocally identified (table 1). Similarly, pair 4 is identifiable except in interchange homozygotes where the $1^{2}$ pair has the same morphology. The chiasma frequencies of pairs 3 and 4, assessed in 54 and 65 individuals respectively, are not correlated with overall chiasma frequency, thus any chiasma increase between classes must be attributable to the rearrangements themselves.

An exact test is provided by the three classes interchange heterozygote $\left(11^{2} 22^{1}\right)$, interchange heterozygote + fission $1\left(1^{\mathrm{s}} 1^{1} 1^{2} 22^{1}\right)$, interchange heterozygote + fission $1^{2}\left(11^{2 \mathrm{~S}} 1^{2 \mathrm{~L}} 22^{1}\right)$. A single structural change distinguishes these three classes: regions 4 and 5 of the pachytene association may be associated or divided by centric fission.

Fission/interchange heterozygotes exceed standard interchange heterozygotes by 0.395 (chromosome 1 fission) and 0.575 (chromosome
$1^{2}$ fission) (table 4). Within the multiple, regions 1,2 and 3 have identical chiasma frequencies in the three classes and the chiasma difference is localised to regions 4 and 5 which span the structural change. In fission heterozygotes, region 4 has about 0.8 chiasmata compared to 0.4 in non-fission heterozygotes. The change in region 5 is less dramatic. In interchange heterozygotes the frequency is 0.78 which rises to 0.92 after fission (table 4; fig. 10).

Regions 4 and 5, then, account for all the difference in chiasma frequency associated with structural change in these three classes. The inference may be drawn that the chiasma differences found between all karyotype classes are similarly localised to the regions affected by rearrangement.

\section{DISCUSSION}

Examination of the chiasma properties of this single family segregating for both centric fission and interchange has revealed three features: (a) the extent of the chiasma increase attributable to the rearrangement is independent of the nature of that rearrangement. Both changes lead to an increase in chiasma frequency of about 0.56 per PMC. (b) these chiasma increases are independent and act together in a simple additive fashion. (c) the increase in chiasma number is localised to the chromosome regions which have been separated into independent pairing units as a result of the rearrangement. There is no effect on other regions of the multiple or on the unaffected bivalents comprising the remainder of the genome.

Effects of structural rearrangements on chiasma formation may be attributable to two apparently distinct phenomena, interference or pairing modification. Centric fission or exchange of pairing partner in an interchange multiple may lead to a reduction of interference in the vicinity with a concomitant increase in chiasma frequency. Inerference has been widely considered an important factor in the control of chiasma number and distribution (Mather, 1938; Fox, 1973). Interference, however, can only be invoked to explain the presence or absence of the second and subsequent chiasmata; the determination of the initial chiasma clearly cannot be interference-dependent (Mather, 1938). Variation in the strength of interference, however, could determine the overall chiasma frequency with ultimately a reduction to one chiasma per bivalent where interference is maximal. We might expect then that chiasma increase associated with a chromosome rearrange- 
Table 4 Chiasma frequencies in the three interchange heterozygote classes. Chiasmata have been allotted to the five segments within the interchange multiple (see fig. 10), pair 3 and pair 4

\begin{tabular}{|c|c|c|c|c|c|c|c|c|c|}
\hline $\begin{array}{l}\text { Karyotype } \\
\text { class }\end{array}$ & $\begin{array}{l}\text { Interch } \\
1\end{array}$ & $\operatorname{moltip}_{2}$ & $\begin{array}{l}\text { gment } \\
3\end{array}$ & 4 & 5 & $\begin{array}{l}\text { Multiple } \\
\text { total }\end{array}$ & Pair 3 & Pair 4 & $\begin{array}{l}\text { PMC } \\
\text { total }\end{array}$ \\
\hline $11^{2} 22^{l}$ & $0 \cdot 932$ & $0 \cdot 300$ & 0.498 & 0.405 & 0.777 & 2.907 & $1 \cdot 339$ & $1 \cdot 159$ & $5 \cdot 405$ \\
\hline $11^{2 \mathrm{~S}} 1^{2 \mathrm{~L}} 22^{1}$ & 0.910 & 0.335 & 0.445 & 0.920 & 0.920 & $3 \cdot 525$ & $1 \cdot 280$ & $1 \cdot 175$ & $5 \cdot 980$ \\
\hline
\end{tabular}

ment would be maximal in families with chiasma frequencies near the lower limit of meiotic efficiency and would decline with increasing chiasma frequency. Chiasma frequencies of basic homozygotes in the six families segregating for fission and interchange reported in this and the previous study varied from $4 \cdot 703$ to $5 \cdot 380$. The chiasma increase attributable to rearrangement in these families ranged from 0.330 to 0.700 and is not correlated with basic chiasma frequency. Indeed, a family has been found with a chiasma frequency in homozygotes of $4 \cdot 200$, near the lower limit of chiasma frequency, in which the $1 / 2$ interchange heterozygotes show the same chiasma level (Parker, unpublished).

A further feature of these results is that the increase in chiasma frequency is not length-dependent. Both the interchange and the centric fission affect chromosome 1 . The $1 / 2$ interchange roughly bisects chromosome 1 with the break-point in the long-arm, and leads to an increase of 0.573 chiasmata per cell. Dissection of chromosome 1 by centric fission gives a 2:1 split and an increase of 0.563 . When the two are combined the overall increase is 1.053 or 0.527 per segment. It is difficult to reconcile this result with control by interference since the magnitude of the chiasma increase would be expected to be dependent on chromosome length.

Many authors have proposed an intimate relationship between chromosome pairing at zygotene and subsequent chiasma formation at pachytene (Maguire, 1977; Rhoades, 1968). In the most extreme situation chiasmata are limited to certain chromosome regions by localised pairing (Henderson, 1969; Fletcher, 1978). By extension,

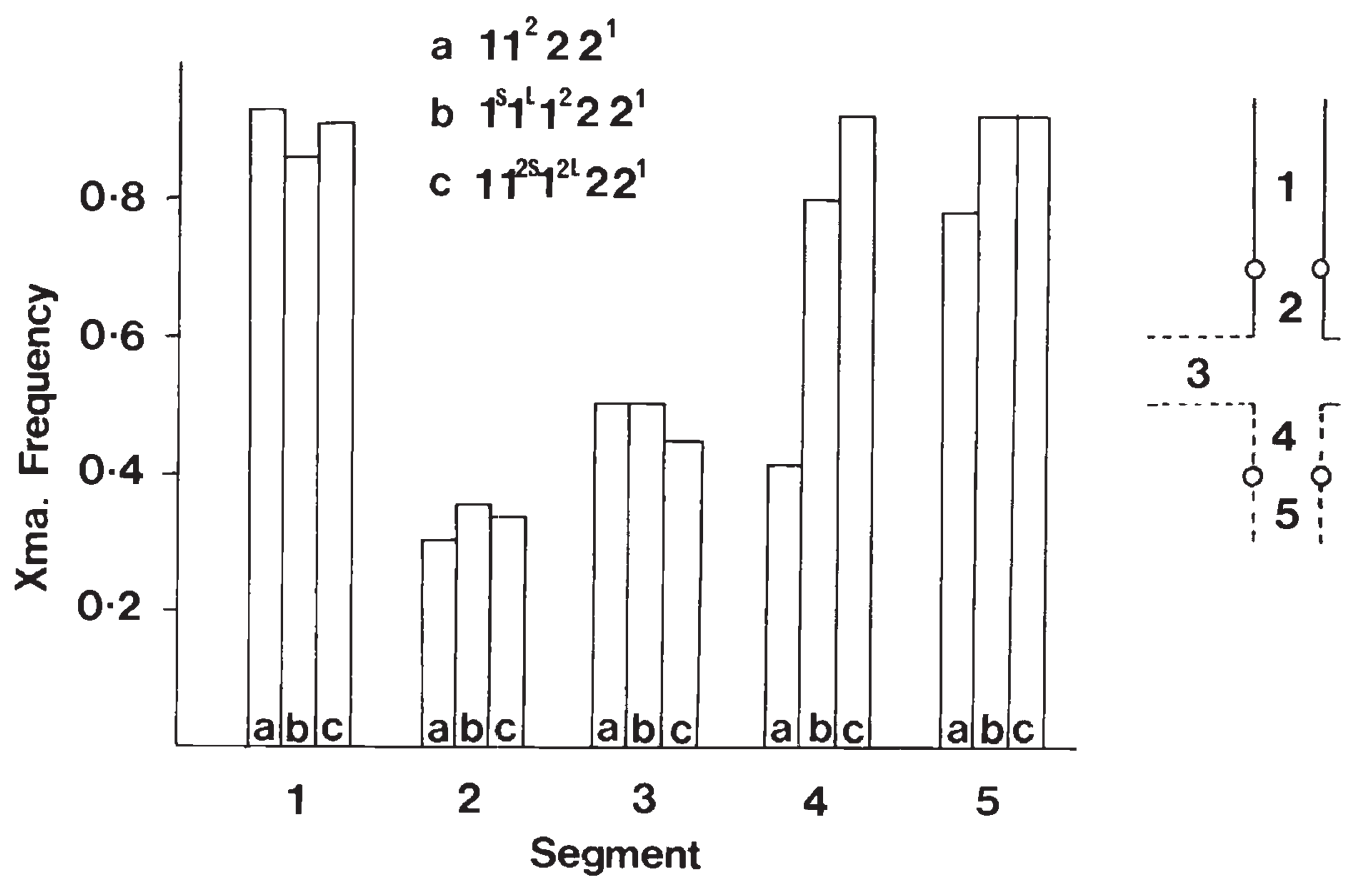

Figure 10 Chiasma frequency distribution in the five segments of the $1 / 2$ interchange multiple. Categories $b$ and $c$ differ from $a$ only in the fission of one or other centromere which separates segments 4 and 5 . The pachytene cross indicating segments 1 to 5 is illustrated. 
it is possible that chiasmata are preferentially located in regions where pairing is initiated and hence is most prolonged. Support for a causal relationship between the progress of pairing and chiasma formation has been obtained by Maguire (1977) in a series of maize inversions and translocation trisomics. Parker et al. (1982) pointed out that in Hypochoeris radicata an increase in the number of potentially-paired regions by chromosome rearrangement was paralleled by an increase in chiasma frequency. The present paper extends these observations and demonstrates that the chiasma increase affects only those regions spanning the pairing discontinuity caused by the rearrangement. It is possible that the alteration in pairing pattern requiring additional initiating sites may result directly in an increased chiasma frequency. It is clearly necessary to analyse the progress of zygotene pairing in both standard and rearranged complements which should be facilitated by the surface spreading technique of Counce and Meyer (1973). Jones (1984) has recently reviewed the evidence for a relationship between pairing and chiasma behaviour and found it suggestive but inconclusive. $\mathrm{He}$ argues that more detailed studies of the pairing process in genic and structural chiasma variants such as those reported here in Hypochoeris are required to explore the interactions of these processes.

\section{REFERENCES}

COUNCE, S. J. AND MEYER, (i. 1. 1973. Differentiation of the synaptonemal complex and the kinetochore in Locusta spermatocytes studied by whole-mount electron microscopy. Chromosoma, 44, 231-253.

FLETCHER, H., 1978. Localised chiasmata due to partial pairing: a 3 D reconstruction of synaptonemal complexes in male Stethophyma grossum. Chromosoma, 65, 247-269.

I ()X, D. P. 1973. The control of chiasma distribution in the locust, Schistocerca gregaria (Forskal). Chromosoma, 43, $289-328$.

HENDERSON, S. A., 1969. Chiasma localisation and incomplete pairing. Chromosomes Today, 2, 56-60.

JONES, (;. H., 1984. The control of chiasma distribution. SEB Symp.

LUCCHESI, J. C., 1976. Interchromosomal effects. Ashburner, M. and Novitski, E. (eds.). In The Cienetics and Biology of Drosophila. Academic Press, London.

maciular, M. 1977. Homologous chromosome pairing. Phil Trans. Rov. Soc. B 277, 245-258.

MATHER, K. 1938. Crossing-over. Biol. Rev, 13, 252-292.

PARKER, J. S., 1975. Chromosome-spccific desynapsis in Hypochoeris radicata. (hromosoma, 49, 391-406.

PARKER, J. S., PALMER, R. W., WHITEHORN, M. A. F. ANI EDGAR, L. A. 1982. Chiasma frequency effects of structural chromosome change. Chromosoma, 85, 673-686.

RHOADFS, M. M. 1968. Studies on the cytological basis of crossing-over. Peacock, W. J. and Brock, R. D. (eds). Replication and Recombination of Genetic Material, Australian Academy of Science, Canberra.

SYBENCiA, 1, 1975. Meiotic configurations. R. Frankel (ed.). In Monographs on Theoretical and Applied Genetics 1, Springer-Verlag, Berlin. 